\title{
Diffusion Engineering by Carbon in Silicon
}

Ulrich Goesele, Pierre Laveant, Rene Scholz, Norbert Engler, and Peter Werner

Max Plack Institute of Microstructure Physics

Weinberg 2, D-06120 Halle, Germany

\section{ABSTRACT}

The possibility to suppress undesirable diffusion of the base dopant boron in siliconbased bipolar transistor structures by the incorporation of a high concentration of carbon has lead to renewed interest in the behavior of carbon in crystalline silicon. The present paper will review essential features of carbon in silicon including solubility, diffusion mechanisms and precipitation behavior. Based on this information the possibilities to use carbon to influence diffusion of dopants in silicon by the introduction of non-equilibrium concentrations of intrinsic point defects will be discussed as well as the reason for the relatively high resilience against carbon precipitation. Interactions between carbon and oxygen will be mentioned, especially in the context of an as yet unexplained fast out-diffusion of carbon close to the surface.

\section{INTRODUCTION}

Besides oxygen carbon is probably the best investigated electrically inactive impurity in crystalline silicon, mostly because carbon is present in appreciable concentrations in silicon after crystal growth. Initially it was of major interest just to characterize its overall behavior in silicon, such as its solubility, diffusion coefficient and precipitation behavior. It was shown that carbon was involved in certain agglomerates of self-interstitials formed during crystal growth of float-zone materials and in certain types of polycrystalline silicon ribbons used for solar cells. It was also noticed that carbon facilitated oxygen precipitation, which had become important for intrinsic gettering purposes. Overall carbon was well investigated and fairly well understood since the middle of the 1980s [1-3]. It was concluded that carbon was a fairly benign impurity which was not really damaging for device processing but did not offer any specific advantages either. Later on it was shown that implanted carbon, in contrast to boron, did not lead to interstitial-type dislocation loops for comparable doses [4]. In addition, implanted carbon showed good gettering capabilities [5] and, at sufficiently high doses, could be used as a very effective etch-stop [6]. Since carbon contracts the silicon lattice considerably it was then investigated carefully as a potential remedy to compensate the lattice expansion associated with germanium in silicon used for heterobipolar transistors [7,8]. For these investigations carbon was incorporated into the silicon lattice during epitaxial crystal growth. Surprisingly, it turned out that carbon can be incorporated in concentrations up to a few percent which is many orders of magnitude above its thermodynamic solubility of carbon in silicon. Most recently carbon incorporation during crystal growth of silicon-germanium alloys was used to induce Si-Ge quantum dot structures [9].

The observation that implanted carbon could reduce the so-called Transient Enhanced Diffusion (TED) of boron after implantation, although only at doses much higher than that of the boron implant, caused some interest in carbon again [5]. This interest in carbon in silicon increased dramatically when it was shown by the group around Gossmann at Bell Labs [10-12] that a relatively high grown-in concentration of carbon practically suppressed TED of boron. This opened up the possibility to fabricate silicon bipolar transistors or Si-Ge based heterobipolar transistors with a very narrow base region (due to the suppressed diffusion of the 
base dopant boron) which is of enormous interest for ultra-high frequency applications [13-16]. Although initially there were concerns that a high concentration of carbon might induce electrically active defects which might deteriorate the device performance, this turned out to be not the case. Initially, the effect of the suppression of boron diffusion was simply interpreted and modeled as a trapping of implantation-induced silicon self-interstitials, which are the diffusion vehicles of boron, by carbon atoms. Later on it was shown by our group at MPI Halle that the effect could be understood and modelled by carbon-diffusion induced undersaturation of silicon self-interstitials [17] and that for a quantitative description besides an undersaturation of self-interstitials also a supersaturation of vacancies has to be taken into account [18]. This way it has become possible to model many aspects of diffusion engineering of dopants by the incorporation of carbon consistently.

The present paper will first cover the basic features of the relevant behavior of carbon in silicon and then turn to the influencing of dopant diffusion by the incorporation of carbon and finally discuss open question such as the precipitation behavior of carbon, an unexplained fast out-diffusion effect of carbon close to the silicon surface and the potential role of oxygen.

\section{BEHAVIOR OF CARBON IN SILICON}

\section{Configurations, Solubilities, Diffusion Mechanisms}

Carbon is mostly substitutionally dissolved in $\mathrm{Si}, \mathrm{C}_{\mathrm{S}}$, which is electrically neutral. The solubility or thermal equilibrium concentration of $\mathrm{C}_{\mathrm{S}}$ is given by [19]

$$
C_{s}^{e q}=4 \times 10^{24} \exp \left(-2.3 / k_{B} T\right) \quad \mathrm{cm}^{3}
$$

as shown in Fig. 1. In contrast to interstitially dissolved oxygen, carbon contracts the silicon lattice as much as the volume of about one atomic volume of silicon for each carbon atom $[20,21]$.

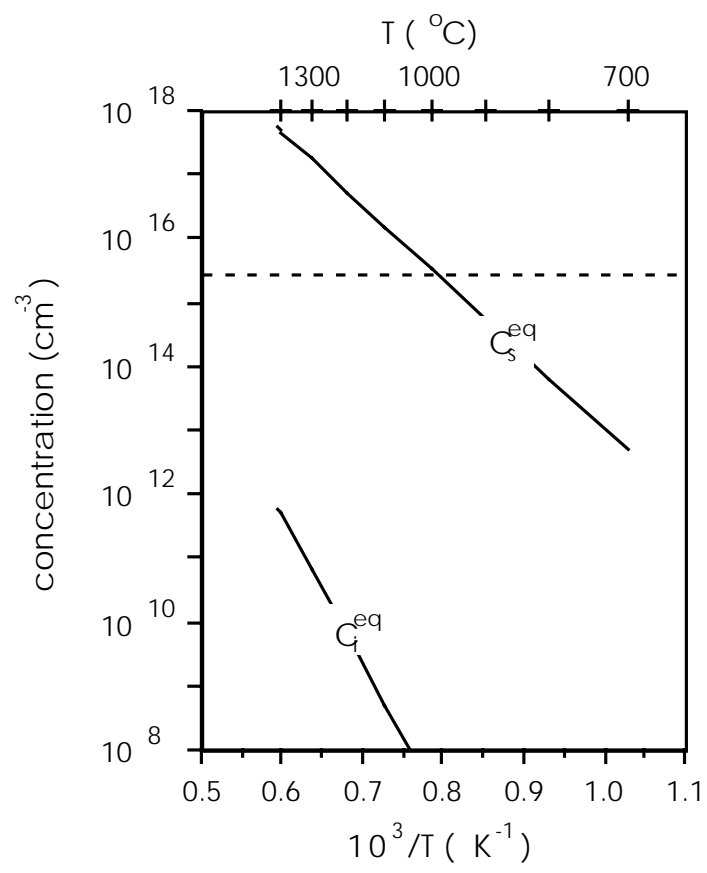

Figure 1. The solubility of substitutionally and interstitially dissolved carbon in $\mathrm{Si}$, denoted as $\mathrm{C}_{\mathrm{s}}^{\mathrm{eq}}$ and $\mathrm{C}_{\mathrm{i}}^{\mathrm{eq}}$, respectively, and given by eqs. (1) and (11), respectively. The dashed line denotes the carbon detection limit by infrared absorption spectroscopy. 


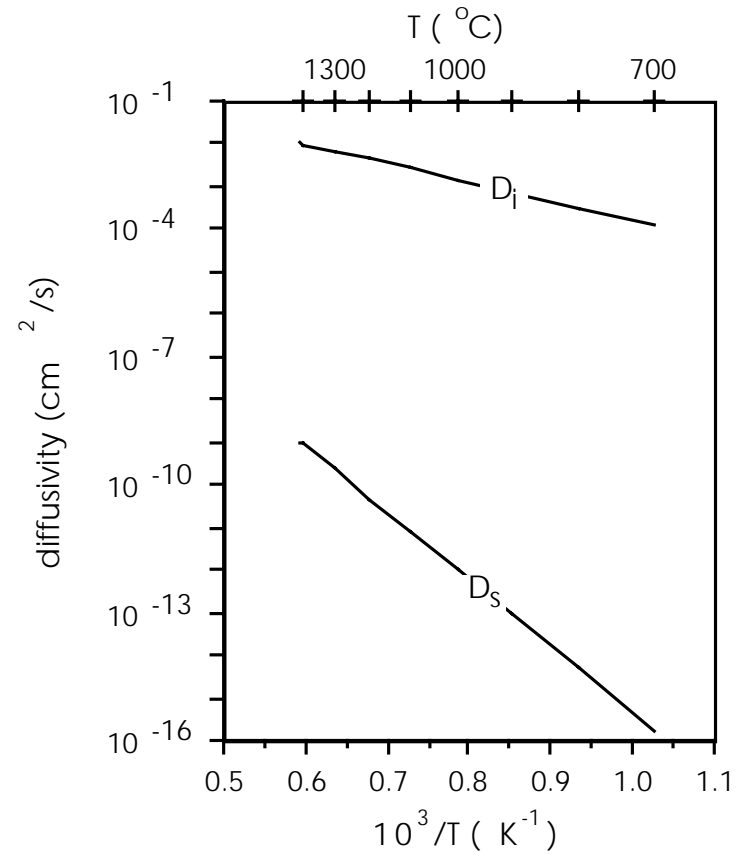

Figure 2. The diffusivities of the substitutionally and interstitially dissolved carbon in $S i$, denoted as $D_{S}$ and $D_{i}$, respectively, and given by eqs. (3) and (2). DS values refer to the case for which the Si point defects are at their thermal equilibrium values.

After electron irradiation at low temperature radiation-induced silicon self-interstitials push carbon atoms from their lattice site forming thereby a carbon-silicon split interstitial, which has been investigated by EPR and infrared absorption [22]. The carbon interstitial, denoted here by $\mathrm{C}_{\mathrm{i}}$, is highly mobile at elevated temperatures based on the diffusion which can be measured at low temperature and is given by $[3,22,23]$

$$
D_{i}=0.44 \exp \left(-0.88 \mathrm{eV} / k_{B} T\right) \quad \mathrm{cm}^{2} / \mathrm{s}
$$

as shown in Fig. 2. The solubility of interstially dissolved carbon can not be measured directly but is accessible via a combination of the diffusion coefficient of substitutional carbon and that of interstitial carbon as described below.

The in-diffusion of substitutional carbon is fairly normal and can be described by a concentration-independent diffusivity leading to an erfc-type concentration profile [1,24,25]. The measured diffusivity of substitutional carbon is a bit higher than that of boron, however, orders of magnitude lower than the measured diffusivity of interstitial carbon (see Fig. 3).

$$
D_{s}=1.99 \exp \left(-3.1 \mathrm{eV} / k_{B} T\right) \quad \mathrm{cm}^{2} / \mathrm{s} .
$$

This is not really astonishing since the diffusion of substitutional dissolved elements usually occurs indirectly by a mechanism involving either vacancies or self-interstitials. In crystalline silicon a number of diffusion mechanisms are involved in self- and impurity diffusion processes [26]. The dominating process depends on the specific element diffusing as well as on other parameters such as temperature, Fermi level, and the presence of supersaturations or undersaturations of intrinsic point defects. We mention in short the various diffusion mechanisms: i) The direct interstitial mechanism holds for interstitially dissolved impurities such as many metallic impurities which act as contaminants or is also valid for interstitial carbon. The diffusivity is extremely high compared to that of substitutionally dissolved atoms such as silicon itself, the common dopants and substitutional carbon. ii) The vacancy exchange 
mechanism involves the exchange of the diffusing substitutionally dissolved atom and a vacancy. In its pure form it only holds for self-diffusion via vacancies in which there is no interaction between vacancies and a silicon atom itself. iii) The vacancy pair diffusion mechanism takes over as soon as an attractive interaction between vacancies $V$ and substitutionally dissolved atoms $\mathrm{A}_{\mathrm{S}}$ occurs and fast diffusing pairs $\left(\mathrm{A}_{\mathrm{S}} V\right)$ form according to

$$
A_{s}+V \Leftrightarrow\left(A_{s} V\right)
$$

iv) The interstitialcy mechanism or the indirect interstitial mechanism refers to the pushing out of a substitutionally dissolved atom by a self-interstitial and its incorporation onto a neighboring substitutional site. v) In analogy to the vacancy pair diffusion mechanism, a fast diffusing pair of a self-interstitial and a substitutionally dissolved atom may form, which more conveniently may then be considered as a special configuration of an interstitially dissolved form $\mathrm{A}_{\mathrm{i}}$ of the impurity atom considered. This directly leads to the so-called kick-out mechanism [27]

$$
A_{s}+I \Leftrightarrow A_{i}
$$

The kick-out mechanism is one of the substitutional-interstitial diffusion mechanisms which governs the diffusion especially of $\mathrm{Au}, \mathrm{Pt}$, and $\mathrm{Zn}$ and leads for the case of very fast diffusing $\mathrm{A}_{i}$ and a sufficiently high interstitial solubility to peculiar in-diffusion profiles characteristic for the kick-out mechanism [26-28]. vi) The Frank-Turnbull [29] or dissociative mechanism is the other substitutional-interstitial diffusion mechanism which involves vacancies and may be described by

$$
A_{s} \Leftrightarrow A_{i}+V \text {. }
$$

The Frank-Turnbull mechanism dominates in the diffusion of $\mathrm{Au}, \mathrm{Pt}$ and $\mathrm{Ni}$ for sufficiently low temperatures at which the kick-out diffusion is kinetically hampered.

The mechanism, which is actually realized for a specific element may either be derived from characteristic profile shapes as in the case of the kick-out mechanism for $\mathrm{Au}, \mathrm{Pt}$ and $\mathrm{Zn}$ or indirectly by the influence of point defect non-equilibrium concentrations on its diffusivity $[26,30]$. In the context of carbon diffusion in silicon, it is interesting that the diffusivity of substitutionally dissolved elements may be enhanced by a self-interstitial supersaturations or an associated vacancy undersaturation, as e.g. generated by surface oxidation or ion implantation of substitutionally dissolved elements. This is expected not only in the case of the interstitialcy mechanism and the kick-out mechanism as expected, but also in the case of the Frank-Turnbull mechanism. We group all these mechanisms together as interstitial-related mechanisms. For the case of substitutionally dissolved elements such as all common dopants their diffusivity $\mathrm{D}_{\mathrm{S}}$ under thermal equilibrium point defect conditions may be split in an interstitial-related part $\mathrm{D}_{\mathrm{s}}^{I}$ and a vacancy-related part $\mathrm{D}_{\mathrm{s}}^{V}$ involving either the vacancy exchange mechanism or its extension (the pair diffusion mechanism) according to

$$
D_{s}=D_{s}^{I}+D_{s}^{V} \text {. }
$$

Under point defect non-equilibrium conditions this changes to

$$
D_{s}^{*}=D_{s}^{I} \frac{C_{I}}{C_{I}^{q q}}+D_{s}^{v} \frac{C_{V}}{C_{V}^{e q}}
$$


Under the assumption of local equilibrium between vacancies and self-interstitials

$$
C_{l} C_{V}=C_{I}^{e q} C_{V}^{e q}
$$

in Eq. (8) the vacancy undersaturation or supersaturation may be replaced by self-interstitialrelated quantities. Based on diffusion enhancements or retardations under point defect nonequilibrium conditions it has been concluded $[26,30]$ that under intrinsic conditions the dopants $\mathrm{B}, \mathrm{P}, \mathrm{Al}$, and $\mathrm{Ga}$ diffuse mostly via an interstitial-related mechanisms, arsenic to a similar extend by both interstitial and vacancy-related mechanisms, and $\mathrm{Sb}$ mostly by a vacancy-related mechanism.

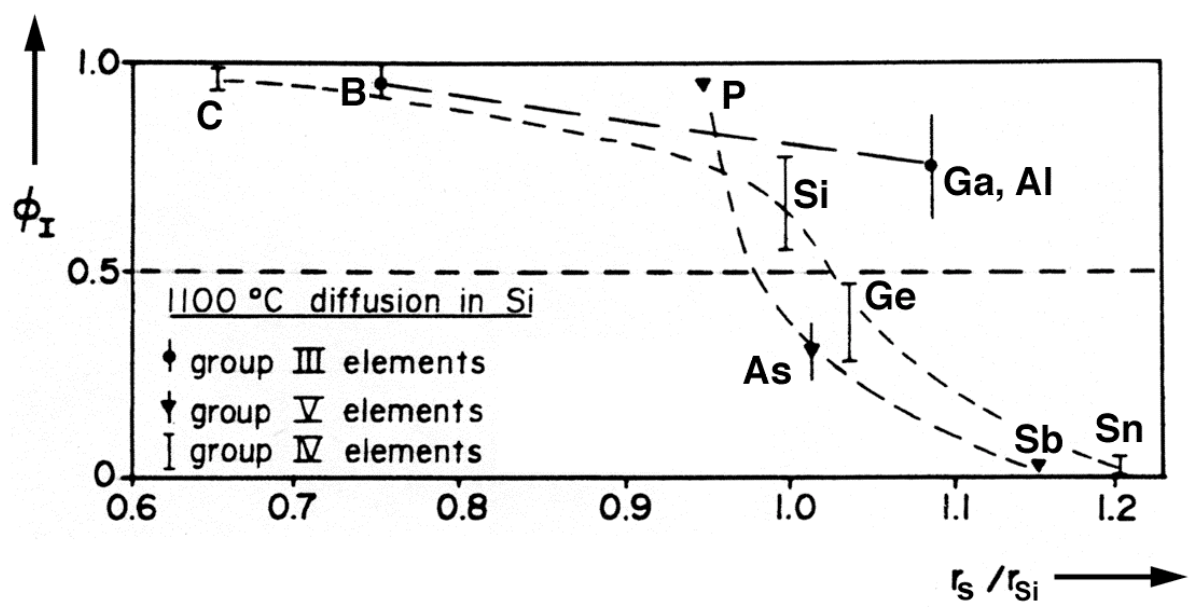

Figure 3. Interstitial-related fractional diffusion component $\Phi_{I}$ for group III, $I V$ and $V$ elements versus their atomic radius in units of the atomic radius $r_{S i}$ for Si.

In the case of carbon, an injection of a supersaturation of self-interstitials by oxidation or high-concentration phosphorus diffusion leads to an enhancement of the carbon diffusion to an extend very similar to that of boron and thus shows no noticeable component via a vacancy exchange or pair mechanism [31]. The normalized interstitial fraction $\Phi_{\mathrm{I}}=\mathrm{D}_{\mathrm{S}}{ }^{\mathrm{I}} / \mathrm{D}_{\mathrm{S}}$ of carbon diffusion is close to one as shown in Fig. 3. Let us mention specifically that these measurements can not and do not distinguish between the kick-out and the Frank-Turnbull mechanism. It turns out that for uncharged substitutionally dissolved atoms such as $\mathrm{C}, \mathrm{Si}, \mathrm{Ge}$, or $\mathrm{Sn}$ the interstitialrelated diffusion component increases with decreasing size. This could be the reason that small carbon atoms diffuse entirely via an interstitial-related mechanism and the large tin atoms mostly via vacancies. Based on the experimental results, substitutional carbon diffusion occurs either via a kick-out or a Frank-Turnbull diffusion process and can be described by

$$
D_{s}=D_{i} C_{i}^{e q} / C_{s}^{e q}
$$

In Eq. (10) the diffusing entity is the interstitial carbon. Since all the quantities except the solubility of interstitial carbon are known experimentally, this allows us to determine the interstitial carbon solubility to be

$$
C_{i}^{e q}=2 \times 10^{25} \exp \left(-4.52 / k_{B} T\right) \quad \mathrm{cm}^{3} .
$$


which is shown in Fig. 1 and turns out to be many orders of magnitude lower than the solubility of substitutionally dissolved carbon. It is worth mentioning that the relatively low diffusivity of substitutional carbon combined with its relatively low solubility prevents the development of a supersaturation of self-interstitials under in-diffusion conditions since the self-diffusion coefficient $D_{I} C_{I}{ }^{\text {eq }}[26,28,30,32]$ is much larger than the product of $D_{i}$ and $C_{S}{ }^{\text {eq }}$. In the presence of a supersaturation of $I$, such as induced by surface oxidation of $\mathrm{Si}, \mathrm{C}_{I}>\mathrm{C}_{I}^{\mathrm{eq}}$ is obtained and the carbon diffusivity simply will be enhanced according to

$$
D_{s}=\left(D_{i} C_{i}^{e q} / C_{s}^{e q}\right)\left(C_{I} / C_{I}^{e q}\right)
$$

An analogous expression holds for boron in the presence of a self-interstitial undersaturation or supersaturation. We specifically mention, that this simple description of carbon diffusion does not hold anymore in the case of the diffusion of a very high concentration of grown-in carbon as used for bipolar transistors.

Precipitation Behavior

The typical concentrations of carbon in $\mathrm{CZ}$ or FZ Si ranges from $10^{15} \mathrm{~cm}^{-3}$ to $5 \times 10^{16} \mathrm{~cm}^{-3}$, but can reach up to $10^{18} \mathrm{~cm}^{-3}$ in deliberately grown C-rich crystals [2] using CZ or FZ methods. By MBE growth, metastable concentrations $C_{S}$ up to $\sim 10^{21} \mathrm{~cm}^{-3}$ can be reached. Considering that the solubility of substitutional carbon is around $10^{15} \mathrm{~cm}^{-3}$ at $1000^{\circ} \mathrm{C}$ it should be expected that carbon present in concentrations orders of magnitude higher than its solubility should precipitate out readily at temperatures at which it can diffuse easily. Experimentally, it turns out that this is not the case [33-35]. Two basic reasons appear to be responsible for this reluctant precipitation. The first reason is the very high interface energy $\sigma_{\mathrm{C}}$ associated with the $\mathrm{SiC} / \mathrm{Si}$ interface which is much higher than the analogous quantity for oxygen precipitates [36]. Whereas oxygen precipitation is associated with a volume increase of about a factor of two, carbon precipitation, independent of whether a carbon agglomerate or $\mathrm{SiC}$ is formed, involves a volume shrinkage of close to one atomic silicon volume for each carbon incorporated in the Cagglomerate or SiC-precipitate (see Fig. 4). As a consequence, a supersaturation of selfinterstitials is supporting carbon precipitation as is well known experimentally, whereas an undersaturation of self-interstitials will make carbon precipitation more difficult $[3,36]$. Formally, this may be expressed by a critical radius $r_{c r i t}$ of such a precipitate

$$
r_{\text {crit }}=\sigma_{c} \Omega / k T \ln \left(C_{S} C_{I} / C_{S}^{e q} C_{I}^{e q}\right)
$$

which does not only depend on the carbon supersaturation but also on the supersaturation or undersaturation of self-interstitials [3].

Within the so-called "+1 model" of implantation of substitutional impurities one expects that each implanted atom generates one net silicon self-interstitial when it gets incorporated on a substitutional lattice site. These self-interstitials may then form interstitial-type dislocation loops. The fact that implanted carbon does not lead to interstitial-type dislocation loops [4] indicates that carbon atoms somehow 're-absorb' the produced self-interstitials. The additional observation that carbon implantation may even reduce TED of boron indicates that carbon may capture back more than just the one extra self-interstitial generated, but roughly ten to fifteen percent more, if the actual results are taken into account [12]. In analogy to the popular "+1 model" one could speak of a "minus $\mathbf{0 . 1}$ model" in the case of carbon implantation. Since the formation of $\mathrm{SiC}$ can account for only one silicon self-interstitial absorbed based on the 


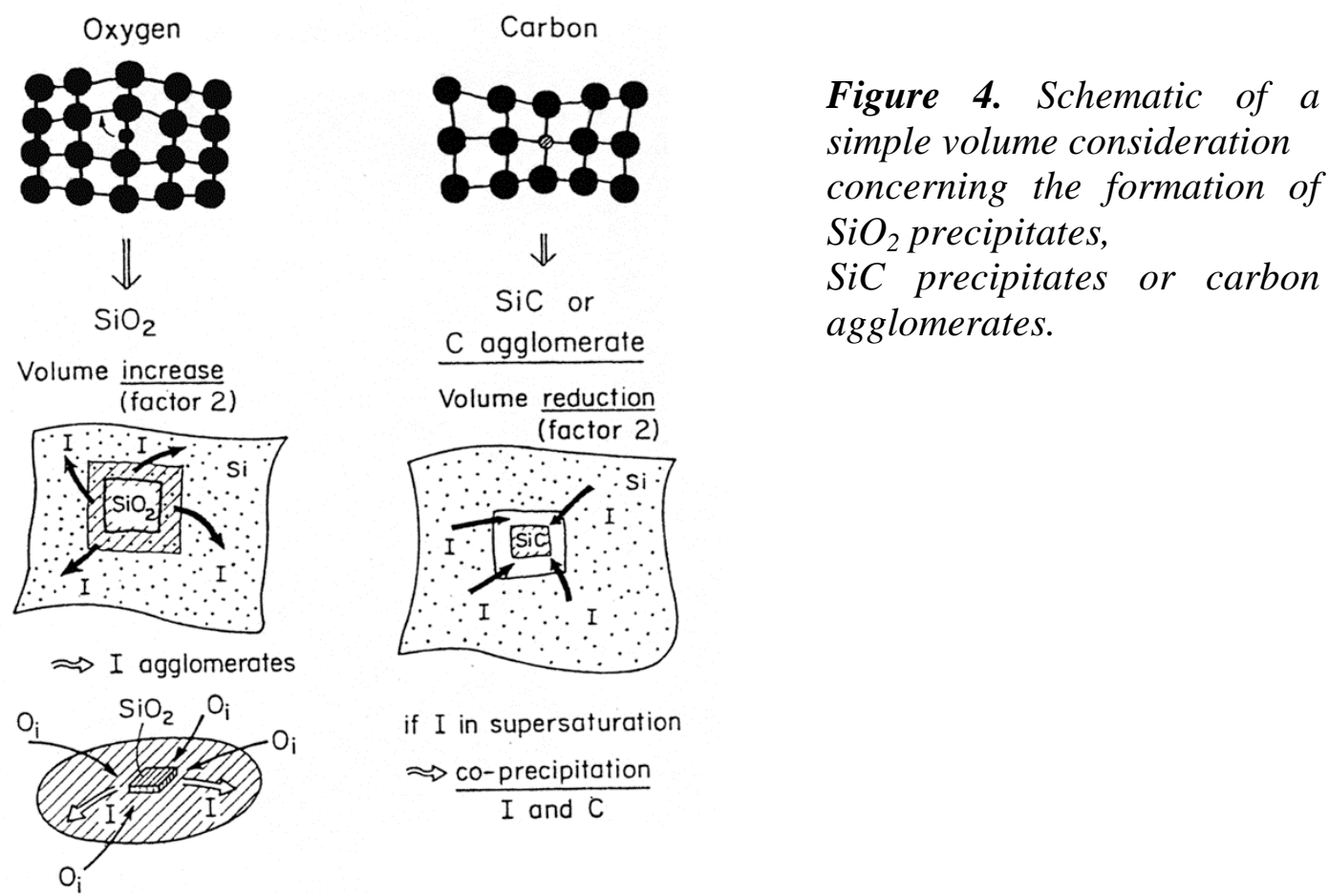

available volume shrinkage, we have to conclude that the initial carbon agglomerate does not consist of crystalline $\mathrm{SiC}$ but rather of some kind of phase which potentially offers a lower interface energy than $\mathrm{SiC}$ (as suggested and observed in the case of metals as well [37]). This initial agglomerate phase should have about a 10-15\% lower specific volume per carbon atom than $\mathrm{SiC}$. In fact, it has been observed that for a sufficiently long precipitation time $\mathrm{SiC}$ forms and that this transformation to $\mathrm{SiC}$ is associated with a release of silicon self-interstitials as expected. Whereas substitutional carbon in silicon is electrically neutral, interstitial carbon or complexes between interstitial and substitutional carbon may be electrically active [24]. Carbon precipitates may also show a certain electrical activity due to interface states as it is well-known from carbon precipitates in carbon rich silicon solar cell material.

\section{Oxygen Effects}

It is immediately obvious that the co-precipitation of carbon (associated with a volume shrinkage) and oxygen (associated with a volume expansion) is favorable and will lead to a coprecipitation in the ratio of two oxygen atoms for each carbon atom precipitated, as experimentally observed $[38,39]$. It appears that oxygen and carbon also interact on an atomic level. It was observed that the out-diffusion of oxygen as well as of carbon is enhanced in the case of the presence of high concentrations of interstitial oxygen and substitutional carbon and leads to about the same enhanced diffusivity [40]. It is tempting to speculate that oxygen and carbon form either a molecular $\mathrm{C}_{\mathrm{i}} \mathrm{O}_{\mathrm{i}}$ complex (corresponding to a $\mathrm{CO}$ molecule) or a corresponding $\mathrm{CO}_{2}$ molecule which may be fast diffusing entities in silicon similar as suggested for $\mathrm{O}_{2}$ in silicon [41]. Such a fast diffusing entity may play a significant role in the diffusion behavior of grown-in carbon in silicon as discussed in the next section. 


\section{BEHAVIOR OF GROWN-IN CARBON AT HIGH CONCENTRATIONS}

As mentioned in the introduction, the interest in the behavior of carbon in silicon increased drastically when it was shown that a high concentration of grown-in carbon almost suppressed TED of boron [11,12]. Initially, it was assumed that substitutional carbon would simply trap silicon self-interstitials and thus reduce their concentration. It became clear relatively quickly that based on the diffusion mechanism of carbon,

$$
C_{s}+I \Leftrightarrow C_{i}
$$

the substitutional carbon can not directly trap self-interstitials, but self-interstitials "trapped" by carbon generate highly mobile carbon interstitials. The immobilizing reaction could then be the following:

$$
C_{i}+C_{s}=\left(C_{s} C_{i}\right)
$$

where $\mathrm{C}_{\mathrm{S}}$ is a substitutional carbon atom, $\mathrm{C}_{\mathrm{i}}$ a highly mobile interstitial carbon atom, and $\left(\mathrm{C}_{\mathrm{S}} \mathrm{C}_{\mathrm{i}}\right)$ an immobile carbon complex consisting of one $\mathrm{C}_{S}$ and one $\mathrm{C}_{\mathrm{i}}$ atoms [24]. However, it turned out that such a model was not able to describe the observed retardation effects consistently. It was then suggested by Scholz et al. [17] that in a carbon-rich region with a carbon concentration far exceeding the carbon solubility the kick-out reaction would proceed to the right hand side consuming self-interstitials and producing a high supersaturation of carbon interstitials which in turn would diffuse out of this region faster than self-interstitials could diffuse back into the carbon-rich region. In consequence, this would not only lead to an undersaturation of selfinterstitials (explaining the retardation of boron diffusion which also uses self-interstitials as diffusion vehicles) but also to characteristic concentration profile shapes for carbon which deviate strongly from erfc-profiles and show a much slower carbon diffusion than expected from carbon in-diffusion data. The requirement for such a non-erfc diffusion behavior is a sufficiently high carbon concentration so that

$$
D_{i} C_{i}^{*}>D_{l} C_{I}^{e q}
$$

is fulfilled in such a region. This is in contrast to the case of carbon in-diffusion, where eq.(16) is not valid. An example for the prediction for an initially box-shaped carbon profile is shown in Fig.5. In the region of high carbon concentration, a severe undersaturation of self-interstitials can be seen. As demonstrated in this example, the concentration of $I$ is reduced by a factor of about 100. The concentration of carbon interstitials in the carbon-rich region as well as the undersaturation may be estimated from the relation, also indicated in Fig. 5 [17]:

$$
C_{I}^{*} / C_{I}^{e q}=\left(D_{I} C_{I}^{e q} / D_{i} C_{i}^{e q}\right) C_{S}^{e q} / C_{S}
$$

where the superscript * refers to concentration values in the carbon-rich region. A calculation of the expected carbon diffusion behavior assuming the diffusion constant measured for carbon indiffusion is also indicated and would overall lead to a much faster diffusion of carbon and a completely different profile shape. The effect of much slower "out-diffusion" than "in-diffusion" is similar to the situation for zinc or beryllium in GaAs in which in-diffusion is also much faster than out-diffusion. 


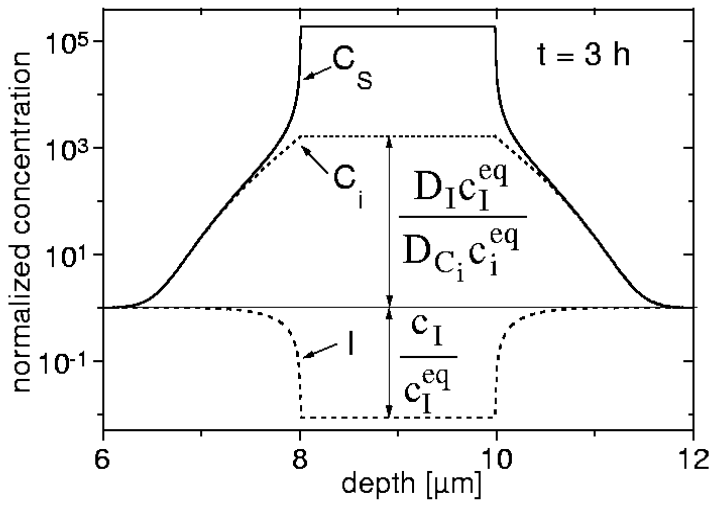

Figure 5. Simulated out-diffusion for a box-shaped carbon profile at $900^{\circ} \mathrm{C}$ for $3 h$ based on the kick-out mechanism. Width and depth of the C box amount to $2 \mu \mathrm{m}$ and $9 \mu \mathrm{m}$, respectively. The associated self-interstitial concentration profile is represented by the dashed line, that of $C_{i}$ by the dotted line, respectively.

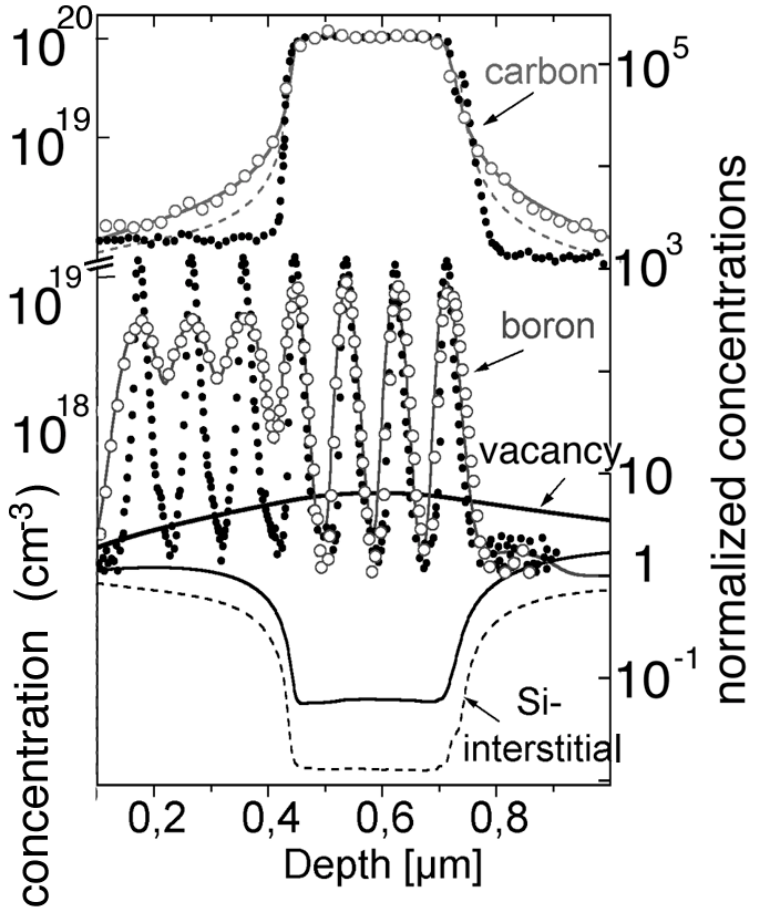

Figure 6. SIMS profiles of a 300nm thick carbon layer with seven boron spikes taken from Rücker et al.[43]. (•) - as grown; (o) annealed at $900^{\circ} \mathrm{C}$ for $45 \mathrm{~min}$; (---) simulated with kick-out mechanism; (-) - with kick-out and Frank-Turnbull mechanism operating at the same time. On the right axis normalized concentration for carbon, $V$ and $I$.

Experimentally, the observed carbon diffusion profile in Fig.6 looks qualitatively very close to what has been predicted based on the kick-out mechanism [15,43,44]. A quantitative fit however requires to take into account also contributions from vacancies via the Frank-Turnbull mechanism [18,35] (Fig. 6). The influence of vacancies reduces the magnitude of the selfinterstitial undersaturation and is associated with a vacancy supersaturation. This extended model, which still has the effective vacancy diffusivity as an adjustable parameter, allows to model the carbon concentration profiles as well as the boron concentration profiles inside and outside the carbon-rich region. The model also predicts that the carbon-induced presence of a supersaturation of vacancies should lead to an increased diffusivity of elements using mainly vacancies as diffusion vehicles such as germanium, arsenic or antimony, as observed experimentally $[15,34,45]$. As mentioned above, the calculated vacancy supersaturation and the associated self-interstitial undersaturation depend somewhat on the effective diffusivity of vacancies. The effective diffusivity of vacancies might be influenced by the presence of interstitial oxygen which may act as a trapping center and releases vacancies in a dynamic equilibrium. It remains to be investigated whether the oxygen concentration may have a noticeable influence on the magnitude of the observed dopant diffusion retardation (for boron and phosphorus [15]) or enhancement (for Ge). 


\section{Near-Surface Effect}

Whereas carbon concentration profiles, which are sufficiently far away (meaning more than about 0.2 micrometers) from the surface, can be modeled nicely by a combination of the kick-out and the Frank-Turnbull mechanism, close to the surface a much higher carbon outdiffusion is experimentally observed than expected according to this model [35]. This higher than expected carbon out-diffusion can phenomenologically be modeled by assuming a higher self-interstitial concentration close to the surface which decays exponentially into the interior. This description is highly unsatisfactory not only because there is no physical reason known which could cause such an exponential decay of an increased self-interstitial concentration close to the surface, but also because this assumed increased self-interstitial concentration should lead to an enhanced boron diffusivity close to the surface. Such an enhanced boron diffusion has not been observed. Therefore, we conclude that a different explanation should be found which does not rely on a changed self-interstitial concentration close to the surface. An attractive possibility appears to be the presence of fast diffusing carbon-oxygen complexes. By considering the reactions between interstitial carbon and interstitial oxygen it can easily be shown that for a constant oxygen concentration level over the whole sample depth this enhanced effective carbon diffusivity should not occur in the regions of high carbon concentration but rather in the regions close to the surface where the $\mathrm{CO}$ molecules may diffuse quickly to the surface and disappear and do not establish a dynamical equilibrium between formation and decay of such $\mathrm{CO}$ molecules. This highly speculative model for the enhanced carbon diffusion close to the surface needs to be checked experimentally and by appropriate computer simulations.

\section{Carbon Precipitation}

The model of a carbon-induced undersaturation of self-interstitials is quite good as long as no carbon precipitation occurs which is not contained in this model. It is obvious and experimentally known that for sufficiently high temperatures and/or sufficiently long annealing times finally carbon precipitation will occur. A simple estimate based on the eq.(13) for the critical radius and the eq.(17) for the undersaturation of silicon self-interstitials in the carbonrich region shows that the undersaturation of self-interstitials drastically increases the critical radius but not to in such an extend that precipitation would be thermodynamically forbidden (since the factor in parenthesis still remains higher than 1 by a factor of more than 100):

$$
r_{\text {crit }}=\sigma_{c} \Omega / k T \ln \left(D_{l} C_{I}^{e q} / D_{i} C_{i}^{e q}\right)
$$

Precipitation should just be strongly delayed and retarded. Since carbon precipitation is likely to lead to electrically active defects, it should thus be avoided during device processing. Therefore, the onset of precipitation should be investigated in more detail experimentally as well as in terms of a reliable simulation model. Based on eq.(18) the following very general conclusions can be drawn: Carbon precipitations becomes more likely for i) higher carbon concentrations, ii) higher temperatures, iii) longer annealing times, iv) larger supply of implantation-induced self-interstitials. In addition, the presence of oxygen certainly facilitates carbon precipitation and will therefore decrease the required time/temperature at which carbon precipitation will occur. The interaction of carbon and oxygen can easily be seen by the increase of oxygen concentration in the area of high carbon concentration as shown in Fig. 7. 


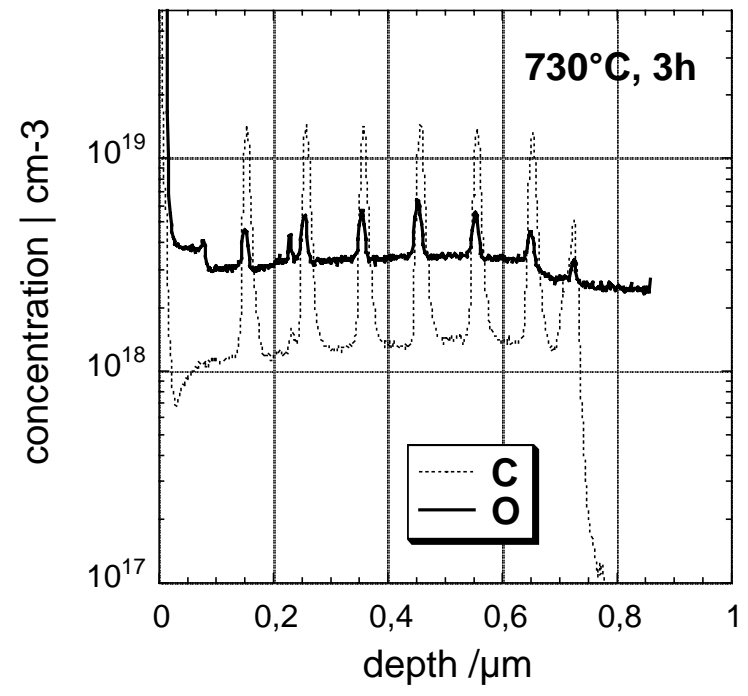

Figure 7. SIMS profile of a superlattice of carbon spikes (width 10nm, distances 100nm, concentration $2 \times 10^{19} \mathrm{~cm}^{-3}$ ) in $\mathrm{Si}$ layer grown by $M B E$. The sample was annealed at $730^{\circ} \mathrm{C}$ for $3 h$.

The corresponding concentration depth profile of oxygen demonstrates its increased concentration within the carbon pikes.

\section{CONCLUSIONS}

The presence of regions of grown-in carbon in concentrations far exceeding its solubility limit leads to an undersaturation of self-interstitials and a supersaturation of vacancies in this region due to the fast out-diffusion of carbon interstitials from this region. On one hand, this effect may be used to decrease the diffusion of dopants such as boron which uses mainly selfinterstitials as diffusion vehicles and to reduce any TED effects of such dopants. This is of high technological importance in the fabrication of bipolar transistors with a narrow base region. On the other hand, the diffusion of dopants or of other substitutionally dissolved elements, such as germanium, diffusing via vacancies will be enhanced. All these effects of diffusion engineering of dopants by the incorporation of a high carbon concentration may be modeled quantitatively as long as no carbon precipitation occurs. This also includes modeling the carbon concentration profiles which smear out much less than expected from the normal carbon diffusivity found in textbooks. There is a clear-cut, which needs to investigate and to model the precipitation of highly supersaturated carbon taking into account the role of self-interstitials, vacancies and oxygen. For understanding the observed fast out-diffusion of carbon close to the surface a highly speculative model based on fast diffusing carbon-oxygen complexes has been suggested which also needs to be checked experimentally.

\section{ACKNOWLEDGEMENTS}

We appreciate close collaboration and discussions with the group of H.J. Gossmann at Bell Labs and with Dr. T.Y. Tan at Duke University in the area of carbon diffusion in silicon.

\section{REFERENCES}

1. $\quad$ R. C. Newman and J. Wakefield, J. Phys. Solids 19, 230 (1961).

2. B. O. Kolbesen, Solid-State Electronics 25, 759 (1982).

3. U. Gösele, Mat. Res. Soc. Symp. Proc. 59, 419 (1986).

4. H. Wong et al., Appl. Phys. Lett. 52, 1023 (1988).

5. W. Skorupa, R.A. Yankov, Mat. Chem. Phys. 44, 101 (1996). 
6. V. Lehmann, K. Mitani, D. Feijoo, and U. Gösele, J. Electrochem. Soc. 138, L3 (1991).

7. W. Faschinger, S. Zerlauth, J. Stangl, and G. Bauer, Appl. Phys. Lett. 67, 2630 (1995).

8. S. C. Jain, H. J. Osten, B. Dietrich, and H. Rücker, Semicond. Sci. Technol. 10, 1289 (1995).

9. O. G. Schmidt, C. Lange, K. Eberl, O. Kienzle, and F. Ernst, Appl. Phys. Lett. 71, 2340 (1997).

10. P. A. Stolk, H.-J. Gossmann, D. J. Eaglesham, D. C. Jacobson, J. M. Poate, and H. S. Luftmann, Appl. Phys. Lett. 66, 568 (1995).

11. P. A. Stolk, H.-J. Gossmann, D. J. Eaglesham, D. C. Jacobson, C. S. Rafferty, G. H. Gilmer, M. Jaraiz, J. M. Poate, H. S. Luftman, and T. E. Haynes, J. Appl. Phys. 81, 6031 (1997); and references there in.

12. N. E. B. Cowern, A. Cacciato, J. S. Custer, F. W. Saris, and W. Vanderhorst, Appl. Phys. Lett. 68, 1150 (1996).

13. L. D. Lanzerotti, J. C. Sturm, E. Stach, R. Hull, T. Buyuklimanli, and C. Magee, Proc. Intern. Device Meeting, Piscataway 1996, p. 249.

14. M.S. Carroll, C.-L. Chang, and J.C. Sturm, Appl. Phys. Lett 73, 3695 (1998).

14. H. Rücker, B. Heinemann, D. Bolze, D. Knoll, D. Krüger, R. Kurps, H. J. Osten, P. Schley, B. Tillack, and P. Zaumseil, Proc. IEDM, Washington, Dec.1999, p.345.

16. H. J. Osten, Carbon-Containing Layers on Silicon, Transtech Publ., Zurich (1999).

17. R. Scholz, U. Gösele, J.-Y. Huh, and T.Y. Tan, Appl. Phys. Lett. 72, 200 (1998).

18. R.F. Scholz, P. Werner U. Gösele and T.Y. Tan, Appl. Phys. Lett. 74, 392 (1999).

19. A.R. Bean, R.C. Newman, J. Phys. Chem. Solids 32, 1211 (1971).

20. J. A. Baker, T. N. Tucker, N. E. Moyer, and R. C. Busher, J. Appl. Phys. 39, 4365 (1968).

21. D. Windish and P. Becker, Phil. Mag. B 58, 435 (1988).

22. G.D. Wattkins and K.L. Brower, Phys. Rev. Lett. 36, 1329 (1976).

23. A. K. Tipping and R. C. Newman, Semicond. Sci. Technol. 2, 315 (1987).

24. G. Davis and R. C. Newman, in Handbook of Semiconductors, vol. 3, 2nd edition, ed. S. Mahajan, p. 1557, North-Holland, Amsterdam 1994.

25. F. Rollert, N. A. Stolwijk, and H. Mehrer, Materials Science Forum 38-41, 753 (1989).

26. W. Frank, U. Gösele, H. Mehrer, and A. Seeger, in: Diffusion in Crystalline Solids, G. E. Murch and A. Nowick, eds. New York, Academic Press, p. 31 (1984).

27. U. Gösele, W. Frank, and A. Seeger, Appl. Phys. 23, 361 (1980).

28. H. Bracht, N. A. Stolwijk, and H. Mehrer, Phys. Rev. B 52, 16542 (1995).

29. F. C. Frank and D. Turnbull, Phys. Rev. 104, 617 (1959).

30. T. Y. Tan and U. Gösele, Appl. Phys. A 37, 1 (1985).

31. J. P. Kalejs, L. A. Ladd, and U. Gösele, Appl. Phys. Lett. 45, 268 (1984).

32. H. Bracht, E.E. Haller, and R. Clark-Phelps, Phys. Rev. Lett. 81, 393 (1998).

33. J. W. Strane, H. J. Stein, S. R. Lee, S. T. Picroux, J. K. Watanabe, and J. W. Mayer, J. Appl. Phys. 76, 3656 (1994).

34. P. Warren, J. Mi, F. Overney, and M. Dutoit, J. Cryst. Growth 157, 414 (1995).

34. R. F. Scholz, P. Werner, P. Laveant, N. Engler, U. Gösele, and H.-J. Gossmann, submitted to J. Appl. Phys.; see also Ph. D. Thesis, R.F. Scholz, Halle 1999.

36. W. J. Taylor, T. Y. Tan, and U. Gösele, Appl Phys. Lett. 62, 336 (1993).

37. E. J. Mittemeijer, personal communication.

38. S. Hahn, M. Arst, K. N. Ritz, S. Shatas, H. J. Stein, Z. U. Rek, and W. A. Tiller, J. Appl. Phys. 64, 849 (1988).

39. Q. Sun, K. H. Yao, J. Lagowski, and H. C. Gatos, J. Appl. Phys. 67, 4313 (1990).

40. F. Shimura, T. Higuchi, and R. S. Hockett, Appl. Phys. Lett. 46, 941 (1985).

41. U. Gösele and T. Y. Tan, Appl. Phys. 28, 79 (1982).

42. $\quad$ S.Yu, T. Y. Tan, and U. Gösele, J. Appl. Phys. 69, 3547 (1991).

43. H. Rücker, B. Heinemann, W. Röpke, R. Kurps, D. Krüger, G. Lippert, and H.J. Osten, Appl. Phys. Lett.73, 1682 (1998).

44. P. Werner, H. Gossmann, D.C. Jacobson, and U. Gösele, Appl. Phys. Lett. 73, 2465 (1998).

45. P. Zaumseil and H. Rücker, Solid-State Phenomena 69-70, 203 (1999). 